\title{
Abordaje de Smith Petersen modificado para fracturas de cuello femoral Pauwels III - Garden IV
}

\section{Modified Smith Petersen Approach to Femoral Neck Fracture Pauwels III - Garden IV}

\author{
Ignácio E. Villalón ${ }^{1}$ Iván Contesse ${ }^{1}$ Cristóbal Vigueras $^{1}$ Javier del Río ${ }^{1,2}$ Julio Piriz ${ }^{2}$ \\ José Antonio Riera ${ }^{1,2}$ \\ ${ }^{1}$ Departamento de Traumatología y Ortopedia, Hospital Clínico \\ Mutual de Seguridad, Santiago, Chile \\ 2 Departamento de Traumatología y Ortopedia, Facultad de Medicina \\ Address for correspondence Ignacio E. Villalón, MD, Departamento de \\ Traumatología y Ortopedia, Hospital Clínico Mutual de Seguridad, Alameda \\ 4848, Santiago, Chile (e-mail: ignaciovillalonm@gmail.com).
} Clínica Alemana de Santiago, Universidad del Desarrollo, Santiago, Chile

Rev Chil Ortop Traumatol 2018;59:117-126.

\section{Resumen \\ Palabras Clave \\ - fractura de cadera \\ - alta energía \\ - Pauwels III \\ - Garden IV \\ El abordaje de Smith Petersen modificado (SPM), permite la reducción de fracturas del cuello femoral desde anterior, manteniendo la fijación a través de un abordaje lateral. Realizar la reducción bajo visión directa mejoraría la calidad de la reducción y los resultados en fracturas complejas de cuello femoral. \\ Métodos Estudio retrospectivo de ocho pacientes con fracturas de cuello femoral Pauwels III/Garden IV secundarias a un accidente de alta energía operadas con abordaje SPM. Registramos el tiempo de evolución desde el accidente hasta la cirugía. Se realizó radiografía y tomografía axial computada postoperatoria. Seguimos los pacientes con radiografías mensuales hasta evidenciar consolidación de la fractura y casos de necrosis avascular. \\ Resultados En promedio, los pacientes fueron operados 2,1 días tras el accidente y fueron seguidos con una mediana de 24,5 meses tras su cirugía. Siete de los ocho pacientes consolidaron. Sólo un caso presentó una no-unión que requirió de una prótesis total de cadera. No hubo casos de necrosis avascular. \\ Conclusión Las fracturas de cuello femoral de alta energía en pacientes jóvenes son lesiones poco frecuentes. Aunque esta serie es pequeña, llama la atención que tratándose de lesiones desplazadas y con rasgos verticales no observamos casos de necrosis avascular, a pesar de algunos casos con prolongado tiempo de evolución desde el accidente hasta la cirugía. Creemos que esas fracturas deben operarse lo antes posible, pero que es más importante lograr una reducción anatómica en vez de retrasar algunas horas la cirugía. En nuestra experiencia, el abordaje de SPM facilita la reducción adecuada en fracturas complejas de cuello femoral.}

Abstract

Modified Smith Petersen Approach (MSP) allows an anterior reduction for femoral neck fractures, keeping the fixation through a lateral approach to the hip. Performing the reduction under direct visualization would improve the quality of the reduction and the outcomes in complex fractures of femoral neck received January 21, 2018 accepted December 13, 2018 ISSN 0716-4548.
Copyright $\odot 2018$ by Thieme Revinter Publicações Ltda, Rio de Janeiro, Brazil 


\section{Keywords \\ - hip fracture \\ - high energy \\ - Pauwels III \\ - Garden IV}

Methods Retrospective study including eight patients with femoral neck fracture classified as Pauwels III / Garden IV, in which MSP was performed. We kept record of the time from the accident until the final surgery. Post-operative studies included plain radiograph and CT scan. Monthly radiographic controls were obtained until fracture healing was achieved or radiological signs of avascular necrosis were found.

Results Surgery was performed in an average of 2.1 days after the accident. Postoperative follow-up was an average of 24.5 month. Consolidation was achieved in seven of the eight patients, only one patient evolved into a nonunion of femoral neck which required a total hip arthroplasty.

Conclusion High energy femoral neck fractures in young patients are infrequent injuries. Even though this is a small series of patients it comes to our attention that being displaced fractures with vertical fracture lines we didn't find any case of avascular necrosis despite some of the cases the prolonged timing since the accident to surgery. We believe that this type of fracture should be treated as soon as possible but is more important to achieve an anatomical reduction than pass over the 12 hours threshold. In our experience MSP approach facilitates the achievement of an adequate reduction and complex femoral neck fractures

\section{Introducción}

Las fracturas de cuello femoral en el paciente joven son lesiones poco frecuentes y por lo general asociadas a traumas de alta energía. ${ }^{1}$ A diferencia de los pacientes mayores, en los jóvenes, el tratamiento de esas lesiones está orientado a preservar la cadera.

Las tasas de complicación publicadas en este tipo de fracturas, incluyendo fallo en la osteosíntesis, pseudoartrosis y necrosis avascular, son altas y varían entre 10 y $45 \%$ de los pacientes. ${ }^{2}$

La reducción anatómica y la fijación interna estable son los objetivos fundamentales del tratamiento quirúrgico ${ }^{3}$ y se consideran como factores claves para lograr buenos resultados. ${ }^{4}$ Por lo general se intenta la reducción cerrada y fijación percutánea, sin embargo, si no se logra una reducción satisfactoria de manera cerrada, se debe realizar una reducción abierta previo a la fijación interna.

El abordaje más frecuentemente utilizado para ese tipo de fracturas ha sido el Watson Jones y Hardinge. ${ }^{5}$ Esos abordajes tienen la ventaja de permitir la reducción de la fractura y la fijación a través de un mismo abordaje, sin embargo, su extensión proximal pone en riesgo el nervio glúteo superior y puede ser difícil el uso de pinzas de reducción desde anterolateral. $^{6}$

En un intento por mejorar la calidad de la reducción, Molnar y Routt ${ }^{7}$ describieron el uso del abordaje de Smith Petersen modificado (SPM) para la reducción de fracturas del cuello femoral, manteniendo la fijación a través de un abordaje lateral (percutáneo o abierto). Ese abordaje sólo usa la porción más distal del abordaje de SP clásico.

A continuación, presentamos nuestra experiencia en el tratamiento quirúrgico de fracturas de cuello femoral Pauwels III/Garden IV a través de un abordaje de SPM.

\section{Material y Métodos}

Estudio retrospectivo - descriptivo de una serie de 8 casos de pacientes con fractura de cuello femoral Pauwels III/Garden IV operadas con abordaje de SPM entre los años 2012 a 2015. La serie fue compuesta por 8 hombres con una mediana de edad de 34 años (22-53 años). Todos los pacientes fueron operados en el mismo centro hospitalario (Hospital de trauma acogido a la Ley del Trabajo) y por el mismo equipo quirúrgico.

Ingresaron al estudio todos los pacientes con presencia de fractura de cuello femoral considerada de alta energía. Se registró mediante el análisis de ficha clínica electrónica el género de los pacientes, edad, mecanismos de lesión y la presencia de lesiones asociadas.

Se analizaron estudios de imágenes digitales en todos los casos. Seis de los ocho pacientes fueron estudiados con radiología simple previo a la cirugía. Todos los pacientes fueron evaluados con TC de cadera para definir el tipo de lesión, y la existencia de rasgos ocultos. En 2 pacientes politraumatizados graves, solo se utilizó el TC.

Todas las fracturas fueron definidas como Pauwels III y Garden $\mathrm{IV}^{8}{ }^{8}$ es decir, rasgos verticales completamente desplazados.

En todos los pacientes con fracturas desplazadas de cuello femoral realizamos intentos de reducción cerrada previo a decidirnos a realizar cirugía abierta. En esos 8 pacientes, consideramos que la reducción cerrada no fue satisfactoria (desplazamiento menor de $2 \mathrm{~mm}$, ausencia de deformidad en varo).

De acuerdo a la técnica quirúrgica descrita por Molnar y Routt, $^{7}$ los pacientes se posicionan oblicuos en una mesa radiolúcida (Orthopaedic Systems Inc, California) sin tracción, para poder mover la pierna libremente. La extremidad inferior ipsilateral a la lesión se incluye completa dentro del campo 

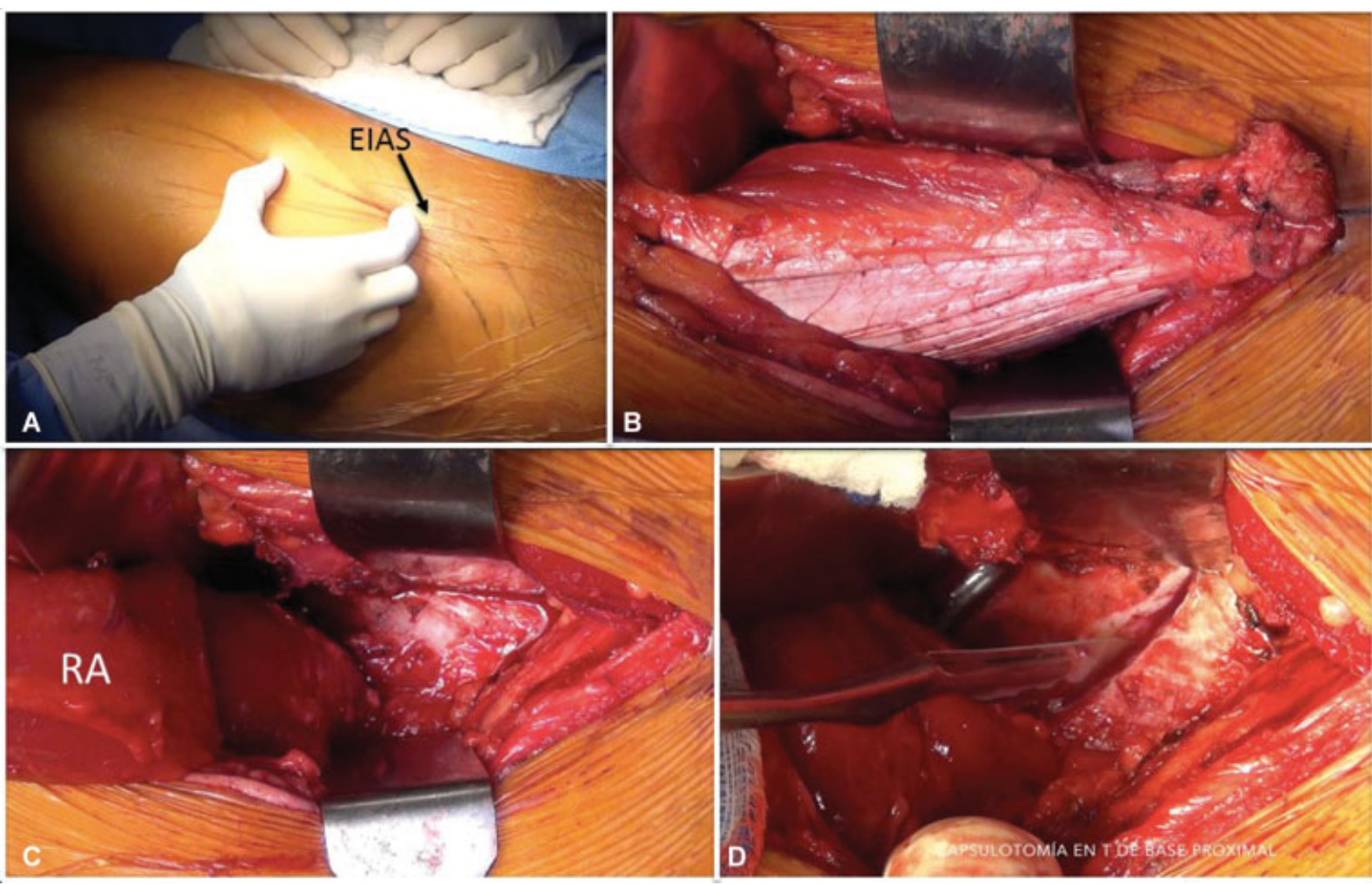

Fig. 1 (A) El abordaje de Smith Petersen Modificado se realiza $2 \mathrm{~cm}$ bajo la espina ilíaca anterosuperior (EIAS) y tiene $10 \mathrm{~cm}$ de longitud. (B) Se identifica el músculo recto anterior en el plano entre el músculo sartorio y el músculo tensor de la fascia lata. Se realiza la tenotomía del m. recto anterior. (C) El músculo recto anterior (RA) se rechaza a distal, con lo cual se expone la cápsula articular de la cadera. (D) Se realiza la capsulotomía en T de base proximal.

operatorio. El arco C se acomoda a la posición del paciente para poder visualizar las proyecciones anteroposterior y axial.

Realizamos un abordaje tipo SPM de $10 \mathrm{~cm}$ de longitud que se inicia $2 \mathrm{~cm}$ bajo la espina ilíaca anterosuperior (EIAS) (-Fig. 1A). Se diseca el celular subcutáneo y se abre la fascia superficial. Se debe identificar y proteger el nervio femoral cutáneo lateral. Se ubica en intervalo entre el músculo sartorio y el tensor de la fascia lata. Profundo a éste se identifica el músculo recto anterior. Se realiza una tenotomía del tendón del recto anterior en la espina ilíaca anteroinferior (EIAI), dejando un remanente tendíneo de $5 \mathrm{~mm}$ aproximadamente para su posterior reinserción. El vientre del músculo recto anterior es desplazado a distal para acceder a la cápsula articular (-Figs. 1B y C). La cápsula se abre mediante una capsulotomía en T oblicua de base proximal que sigue el cuello femoral ( - Fig. 1D). Se drena el hematoma intracapsular. Se identifica el foco de fractura, se limpia de coágulos y partes blandas (sinovial) ( - Fig. 2A) y se realiza la reducción bajo visión directa con pinzas, pueden utilizarse agujas Kirschner en la cabeza femoral a modo de “joystick" (-Figs. 2B y C). Una vez lograda la reducción anatómica se realiza la fijación de la fractura por lateral ya sea de manera percutánea o abierta según el método de osteosíntesis elegido ( - Fig. 2D).

En los casos que se requiera acceder al acetábulo (columna anterior) el abordaje se puede extender a proximal sobre la cresta ilíaca. El abordaje anterior permite utilizar placas en el cuello femoral en casos de encontrar dificultades para mantener la reducción con pinzas.
Una vez realizada la osteosíntesis se retiran las pinzas de reducción.

Cerramos la cápsula con puntos simples. Reinsertamos el músculo recto femoral con flexión de cadera, utilizando sutura reabsorbible resistente (vicryl 1 CT-X).

Todos los casos fueron evaluados con radiografía y TC postoperatorio para evaluar la calidad de la reducción y la posición adecuada de nuestros implantes. Realizamos radiografías mensuales hasta evidenciar consolidación de la fractura.

Nuestro protocolo de manejo postoperatorio es con fisioterapia desde el primer día después de la cirugía y descarga por 8-10 semanas, tras lo cual se inicia la carga progresiva para completar la carga a las 12 semanas de la cirugía.

Además, registramos el tiempo de evolución en horas o días desde el accidente hasta la cirugía y el tiempo de evolución desde la cirugía hasta el último control.

Se siguieron los pacientes con radiografía simple hasta la consolidación de la fractura, registrando los casos de no unión y necrosis avascular.

Se consideró como fracaso de tratamiento quirúrgico la no unión de la fractura o necrosis avascular de la cabeza femoral que requieran prótesis total de cadera.

\section{Resultados}

Seis pacientes sufrieron accidentes de tráfico y dos caídas de altura. 


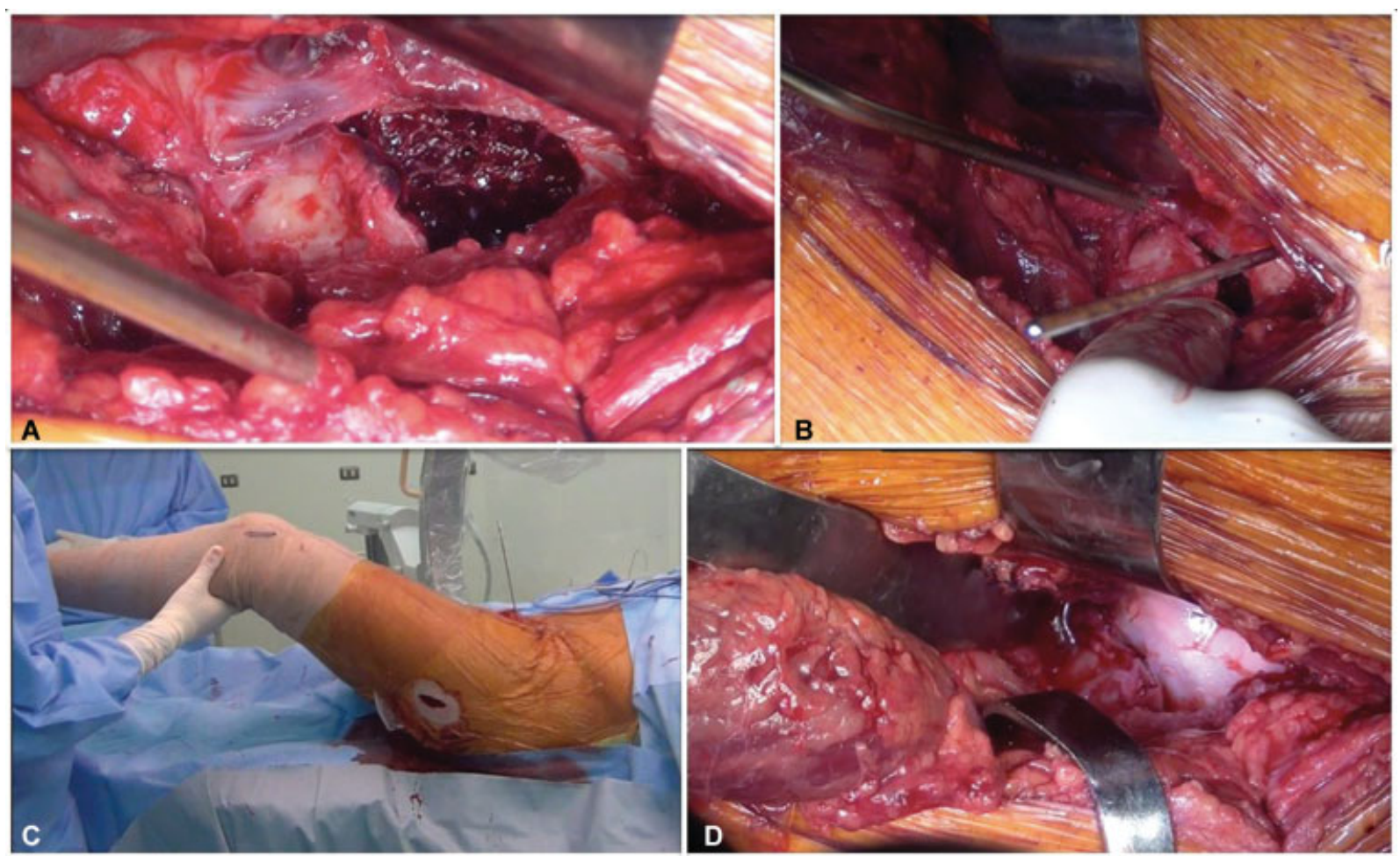

Fig. 2 (A) Se identifica el foco de fractura, que se limpia de sinovial y coágulos. (B) Se realiza la reducción de la fractura bajo visión directa. Se puede utilizar una aguja Kirschner en la cabeza femoral para corregir la rotación. (C) A través de un abordaje lateral se realiza la fijación de la fractura. Nótese que la extremidad inferior esta libre en el campo operatorio. (D) La calidad de la reducción y estabilidad de la osteosíntesis puede verificarse bajo visión directa.

Tres pacientes presentaban la fractura de cuello femoral como lesión aislada. Un paciente presentaba como lesión asociada fractura del fémur ipsilateral y otro paciente fractura de ambos fémures. Los otros tres casos, eran pacientes politraumatizados con múltiples lesiones músculoesqueléticas dos de los cuales incluían fémur ipsilateral.

La lesión asociada más frecuente fue la fractura de fémur ipsilateral (4 casos).

Sólo un paciente en esta serie fue operado antes de las 12 horas (10 horas), 4 pacientes antes de las 24 horas del accidente, un paciente a las 48 horas del accidente, otro a los 4 días y un paciente a los 7 días del accidente.

Como método de osteosíntesis se utilizaron 3 tornillos canulados en 6 pacientes. En un caso se utilizó un DHS más tornillo antirotatorio $7,0 \mathrm{~mm}$ canulado y en otro caso DHS más tornillo canulado $7,0 \mathrm{~mm}$ más placa anterior al cuello femoral $2,7 \mathrm{~mm}$.

Los pacientes fueron seguidos en una mediana de 24,5 meses (6-38 meses) tras su cirugía.

Siete de los ocho pacientes consolidaron en posición anatómica, sin acortamiento ni desplazamiento en varo.

Sólo un caso requirió de una prótesis total de cadera, es decir, hubo 1 fracaso del tratamiento en ese grupo de 8 pacientes. Ese paciente fue operado antes de las 24 horas de evolución con 3 tornillos canulados, sin embargo, rápidamente evolucionó a una falla de la osteosíntesis, con desplazamiento secundario por lo cual fue tratado con una artroplastia total de cadera 4 meses tras su cirugía inicial.

No hubo casos de necrosis avascular.

\section{Discusión}

Las fracturas de cuello femoral de alta energía en pacientes jóvenes son lesiones poco frecuentes, por lo cual es difícil reunir un número de casos que permita reportar una serie. Según un estudio canadiense, la mayoría de los traumatólogos se ve enfrentado a menos de 5 casos de fracturas de cuello femoral en pacientes jóvenes al año con o sin desplazamiento. ${ }^{9}$ Nosotros presentamos ocho casos de fracturas Garden IV y Pauwels III (-Figs. 3 y 6), en que se utilizó el abordaje de SPM, operados por el mismo equipo quirúrgico en un período de 38 meses. Existen pocas series en la literatura como esta.

La dificultad en lograr grandes series de pacientes es una de las causas de que en la actualidad existan muchas incógnitas en el tratamiento ideal de esos pacientes.

Aunque esta serie es pequeña, llama la atención que tratándose de lesiones desplazadas y con rasgos verticales no observamos casos de necrosis avascular, a pesar de algunos casos con prolongado tiempo de evolución desde el accidente hasta la cirugía. Más aún cuando en la literatura se describen tasas de necrosis avascular de hasta $45 \%$ es este tipo de fracturas. ${ }^{10}$ Incluso uno de nuestros pacientes que fue operado 7 días después de su accidente, permaneciendo con un desplazamiento severo por todo ese período de tiempo y consolidó sin inconvenientes (- Figs. 3, 4 y 5). Esos resultados son similares a lo publicado en la literatura recientemente. ${ }^{11}$ Papakostidisy col., ${ }^{3}$ en 2015 realizaron una revisión sistemática de la literatura. No pudieron demostrar asociación entre el 


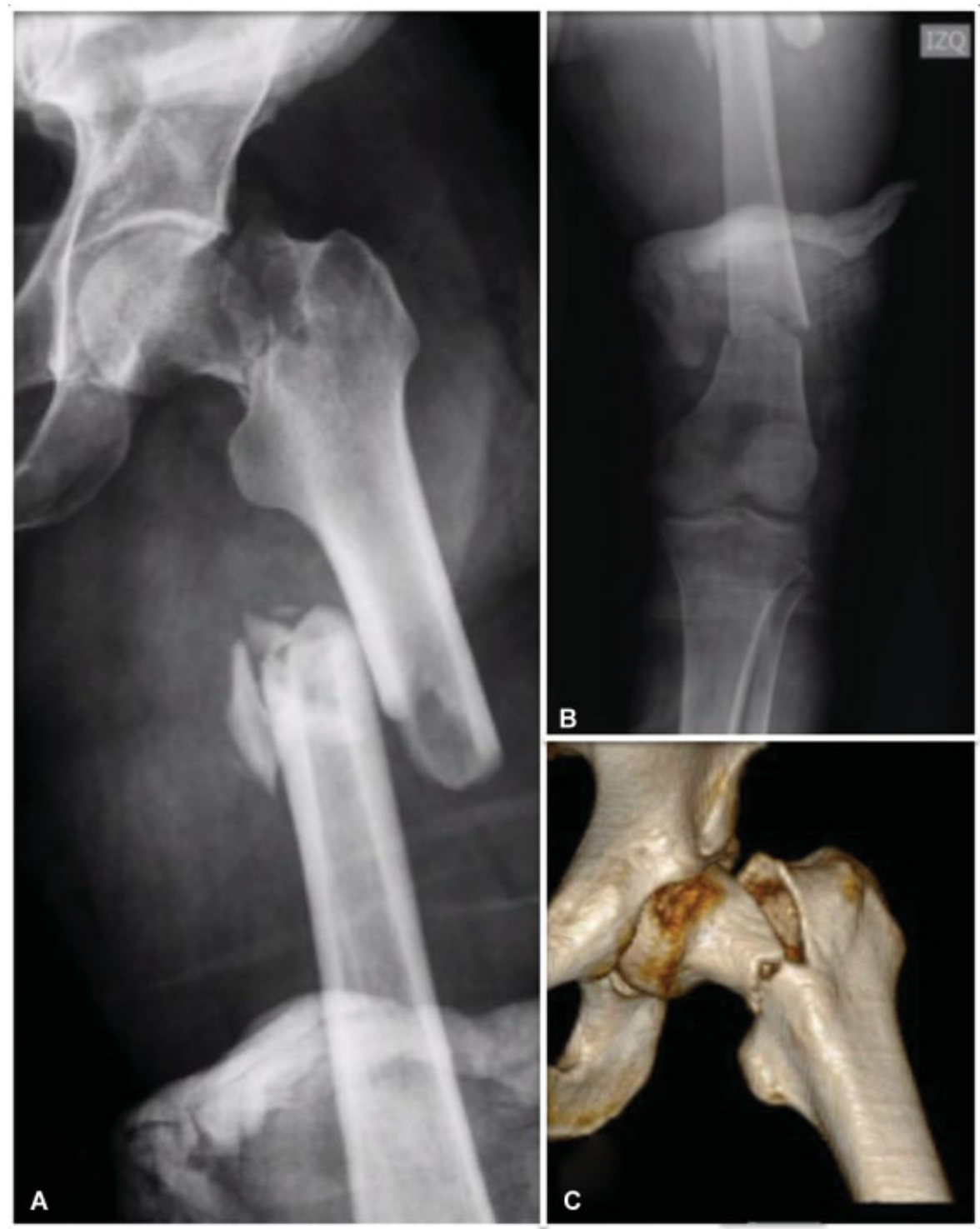

Fig. 3 (A y B) Paciente de 53 años con fractura Pauwels III - Garden IV. (C) El TC preoperatorio demuestra una fractura vertical con desplazamiento completo.

tiempo transcurrido desde el accidente hasta la cirugía con riesgo de necrosis avascular. Pero sí que observaron un aumento de las no uniones en los pacientes operados después de las 24 horas. Aunque el origen de la necrosis avascular en fracturas del cuello femoral probablemente es multifactorial, ${ }^{10}$ nuestra experiencia y algunos artículos recientes se contraponen a los conceptos clásicos de manejo de esas lesiones ${ }^{11,12}$ y nos hacen pensar que más que el tiempo hasta la cirugía, uno de los principales factores que determina el pronóstico es la calidad de la reducción. Como otros autores, creemos que esas fracturas deben operarse lo antes posible, pero que es mejor esperar a tener el equipo adecuado y las condiciones ideales para lograr una reducción anatómica, es decir, probablemente esa debería ser la primera cirugía del día, antes que operar de noche urgente a esos pacientes con el fin de no pasar más allá de las "12 horas" de evolución. ${ }^{11}$

El material de osteosíntesis a elegir también es motivo de controversia. A pesar de que existe una literatura que apoya el uso de dispositivos con ángulo cérvico-diafisario fijo (DHS), lo más frecuentemente utilizado son los tornillos canulados. Tres tornillos son el número adecuado, ya que aumenta la estabilidad versus 2 tornillos. ${ }^{13}$ Aparentemente cuatro tornillos no son más estables que 3. En nuestra experiencia, si la reducción es anatómica, con interdigitación de las espículas óseas y se logra compresión adecuada, los tornillos canulados son suficientes para mantener la reducción incluso en fracturas con rasgos verticales. En casos con conminución, anclaje óseo pobre de los tornillos o evidencia intraoperatoria de inestabilidad, preferimos utilizar un DHS más un tornillo canulado antirotatorio. Además, en casos seleccionados podemos adicionar una placa anterior en el cuello femoral para mantener la reducción (- Figs. 6 y $\mathbf{7}$ ).

En nuestra opinión, de utilizar tornillos canulados algunos aspectos técnicos pueden mejorar los resultados: 1.intentamos posicionar los tornillos lo más periféricos posible 

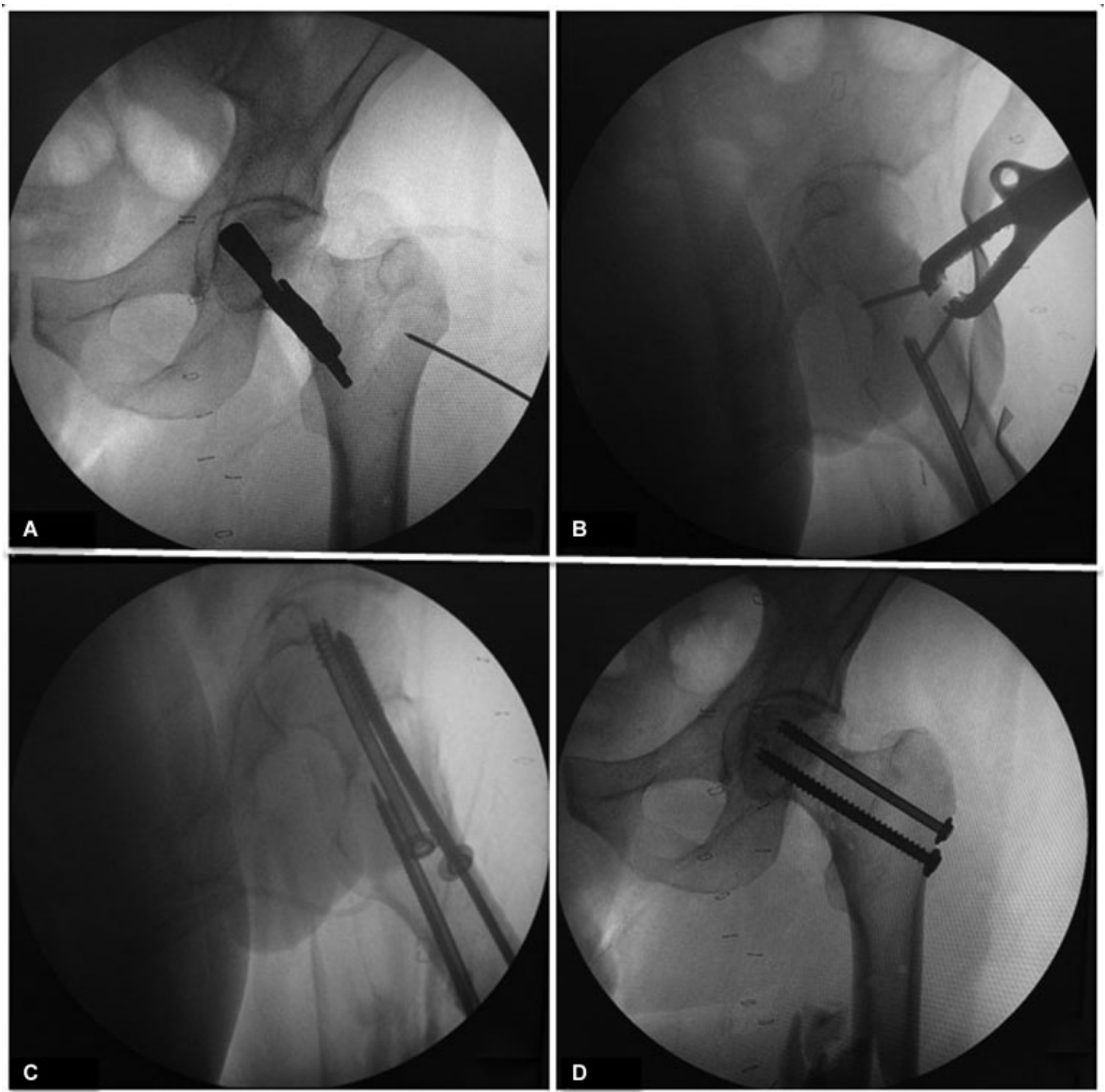

Fig. 4 Secuencia intraoperatoria del paciente de la - Fig. 3. (A) Vista anteroposterior de la reducción desde anterior a través del abordaje SPM con una pinza tipo Farabeuf y 2 tornillos $3,5 \mathrm{~mm}$. (B) Vista axial; tras la reducción anatómica se realiza la osteosíntesis desde lateral con tornillos canulados 7,0 mm. (C) El primer tornillo (superior - central) realiza compresión (rosca $16 \mathrm{~mm}$ ). El segundo tornillo también realiza compresión (inferior - anterior) (D) El último tornillo (inferior posterior) bloquea la compresión (rosca completa). Se logra la reducción anatómica de la fractura. La fractura de la diáfisis femoral se trata a continuación con un clavo endomedular retrógrado.

para que se acerquen a las corticales. Evitamos que los tornillos se aglomeren en la zona central de la cabeza. 2.- inicialmente realizamos compresión con tornillos rosca corta que pasen el rasgo de fractura (16 o $32 \mathrm{~mm}$ ) pero una vez comprimida la fractura utilizamos tornillos rosca completa (segundo $\mathrm{y} / \mathrm{o}$ tercer tornillo) para mejorar el anclaje óseo y evitar el colapso excesivo que a veces se observa al usar sólo tornillos rosca corta. Incluso en ocasiones, tras la instalación del segundo y tercer tornillo cambiamos el primero a un tornillo rosca completa. A pesar de consolidar, si los pacientes jóvenes presentan exceso de acortamiento, el resultado puede ser insatisfactorio para el paciente ya que evolucionan con cojera residual. Esa es una complicación más frecuente de lo que se cree. Stocktony col., ${ }^{14}$ en una serie de 65 pacientes menores de 60 años, observaron un acortamiento severo ( $>1 \mathrm{~cm}$ ) en $32 \%$ de los casos. Eso se traduce en peores resultados en la escala SF-36 de función física. ${ }^{15}$ Otra manera de prevenir el acortamiento y aumentar la estabilidad es utilizar una placa anterior en el cuello femoral. 3.- habitualmente utilizamos 3 tornillos en delta de base distal para evitar el tornillo en la zona superior posterior, ya que en esa zona se encuentran los vasos que irrigan la cabeza femoral. 


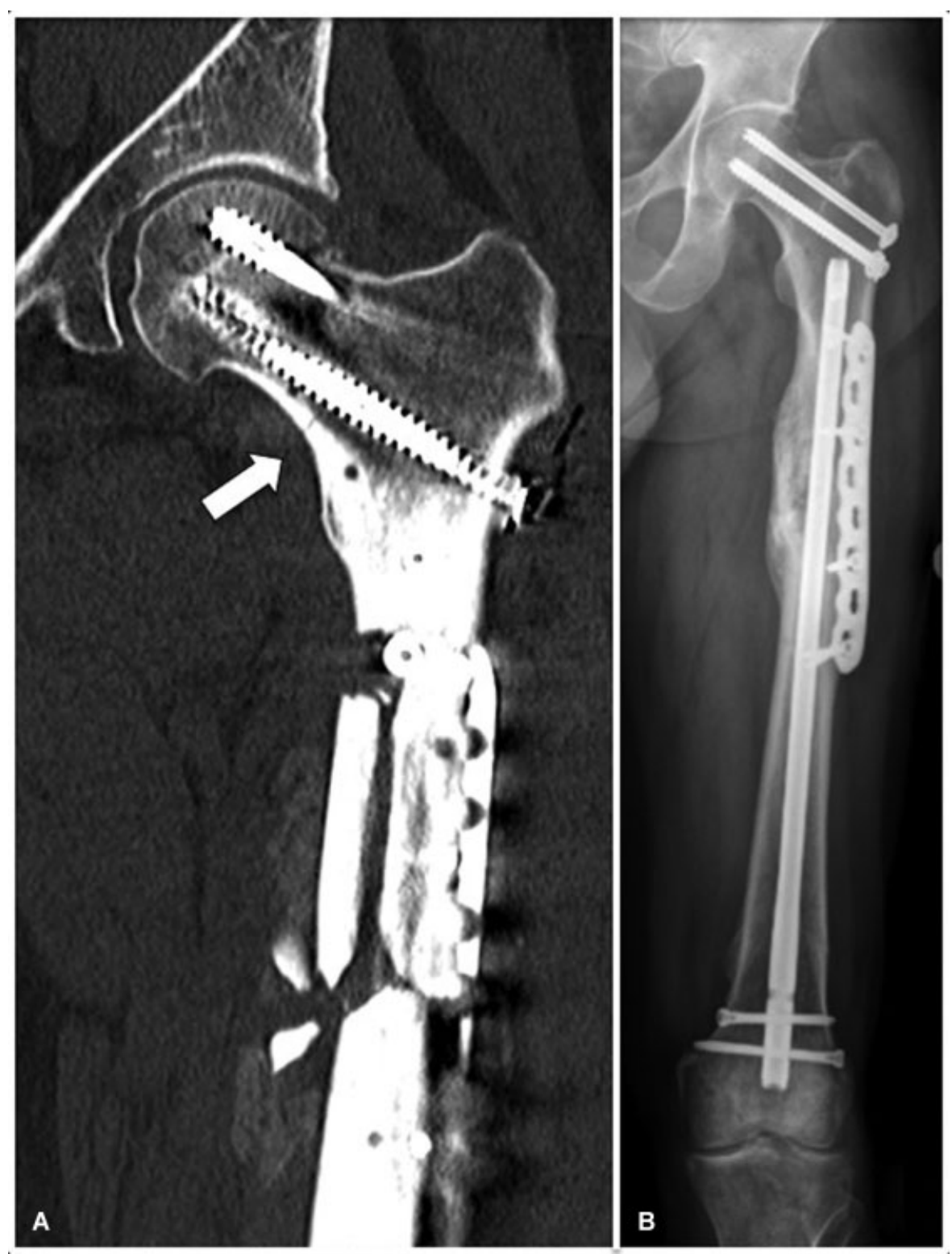

Fig. 5 (A) TC a las 6 semanas de evolución demuestra la reducción anatómica de la fractura (flecha) y la adecuada posición de los tornillos. Se observa callo inicial en la fractura de fémur. (B) Radiografía de control al año con las fracturas consolidadas, sin evidencias de necrosis avascular de la cabeza femoral.

Cuatro pacientes (la mitad de la serie) presentaban fractura de fémur ipsilateral. Incluso en un caso era segmentaria (-Figs. 3, 4 y 5). El abordaje SPM de gran utilidad en pacientes con fractura de cuello femoral asociada a fractura de diáfisis femoral cuando las dos fracturas presentan desplazamiento importante, ya que permite reducir de manera anatómica y fijar la fractura del cuello aunque la diáfisis no esté aún fija. Tras tratar el cuello femoral, se puede tratar la diáfisis de manera independiente. Intentar la reducción y osteosíntesis de ambas fracturas con un solo implante (clavo céfalo-medular), si ambas están desplazadas es técnicamente complejo. La combinación SPM con tornillos canulados más clavo femoral retrógrado, se ha transformado en nuestro tratamiento de elección para ese tipo de pacientes si presentan desplazamiento importante de su fractura de cuello y diáfisis femoral.

El abordaje de SPM permite verificar la calidad de la reducción bajo visión directa y utilizar pinzas e implantes por anterior sin dificultar la fijación interna por lateral. Realizado correctamente no implica riesgo de lesiones vasculares o neurológicas a diferencia del Watson Jones en su extensión proximal (nervio glúteo superior). Otra de las ventajas del SPM es que en caso de fallo de la osteosíntesis, no unión o necrosis avascular, no compromete los abordajes clásicos a través de los cuales se realizan las artroplastias de cadera. $^{7}$ La desinserción del recto anterior es una de sus desventajas. Aunque es fácil su reinserción al finalizar el procedimiento quirúrgico se desconoce su real morbilidad. ${ }^{16}$ 

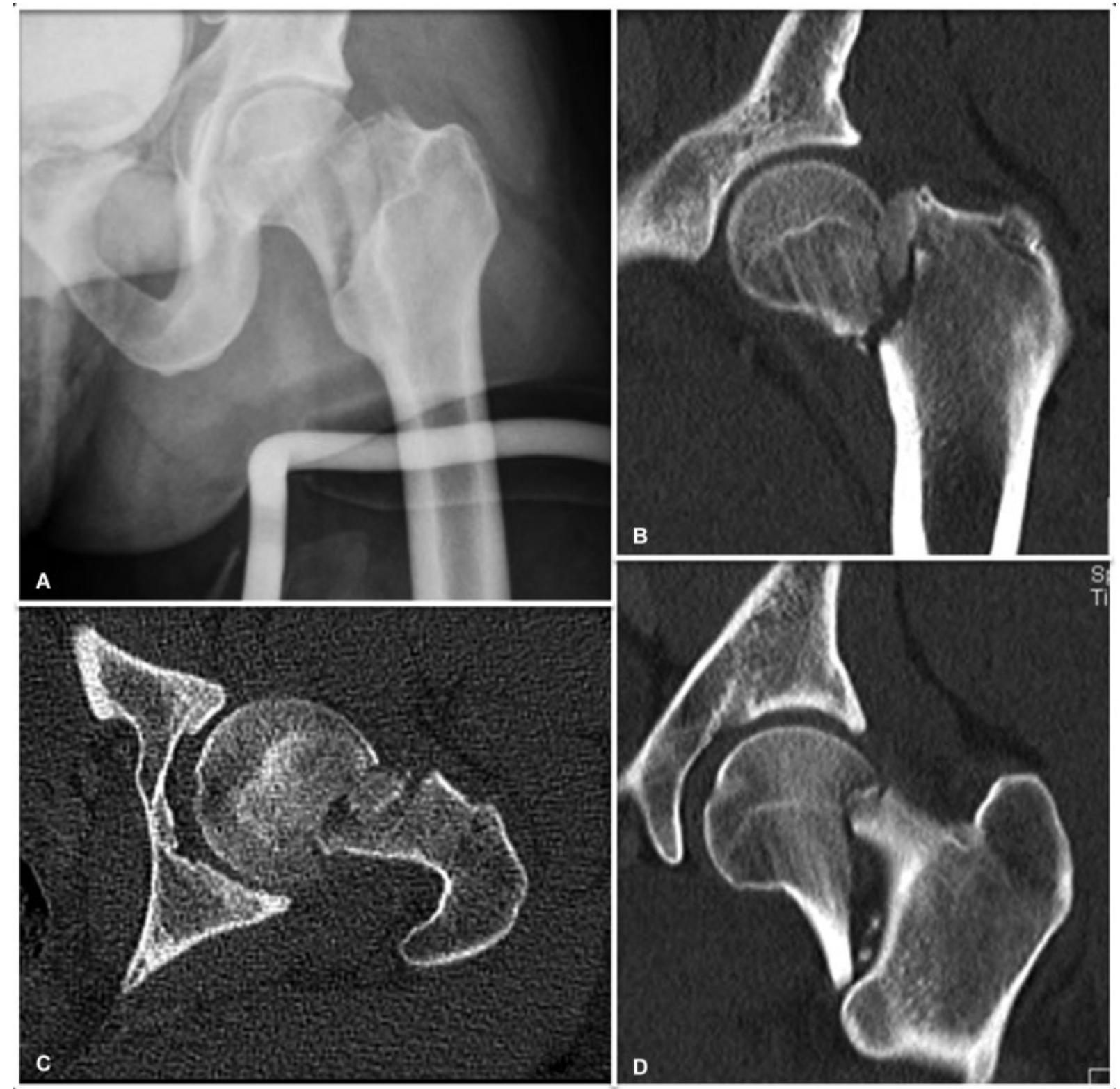

Fig. 6 (A) Paciente de 22 años con fractura Pauwels III - Garden IV de 4 días de evolución. (B, Cy D) El TC preoperatorio demuestra una fractura vertical con desplazamiento completo del cuello femoral. Se observa conminución con un fragmento intermedio.

Ye y col., ${ }^{17}$ en una serie de 28 pacientes con fracturas de cuello femoral Pauwels III, realizaron una reducción y osteosíntesis abierta mediante abordaje anterior directo con 3 tornillos canulados asociado a una placa "buttress" medial. A corto plazo, obtuvieron un $89 \%$ de consolidación sin acortamiento del cuello femoral. En 3 casos, observaron falla del implante. Concluyen que mediante la visualización directa, se permite obtener una reducción anatómica y asociar el uso de placas sin agregar morbilidad.

Dentro de las fortalezas de este estudio, cabe mencionar que es el primer reporte nacional de ese tipo de lesiones tratadas mediante reducción abierta y fijación interna. Si bien es sólo un reporte de 8 casos con buenos resultados a corto y mediano plazo, puede marcar el tratamiento de esas fracturas en ese grupo de pacientes. Dentro de las debilidades, corresponde a un estudio retrospectivo con un seguimiento a corto y mediano plazo.

\section{Conclusión}

Este abordaje es una herramienta útil para lograr reducciones anatómicas abiertas en caso de no lograrse con maniobras cerradas. Los resultados radiológicos en esta serie se consideran buenos para el manejo de ese tipo de fracturas.

\section{Conflicto de Intereses}

Los autores del presente trabajo no refieren ningún conflicto de interés con ninguna entidad pública o privada. 


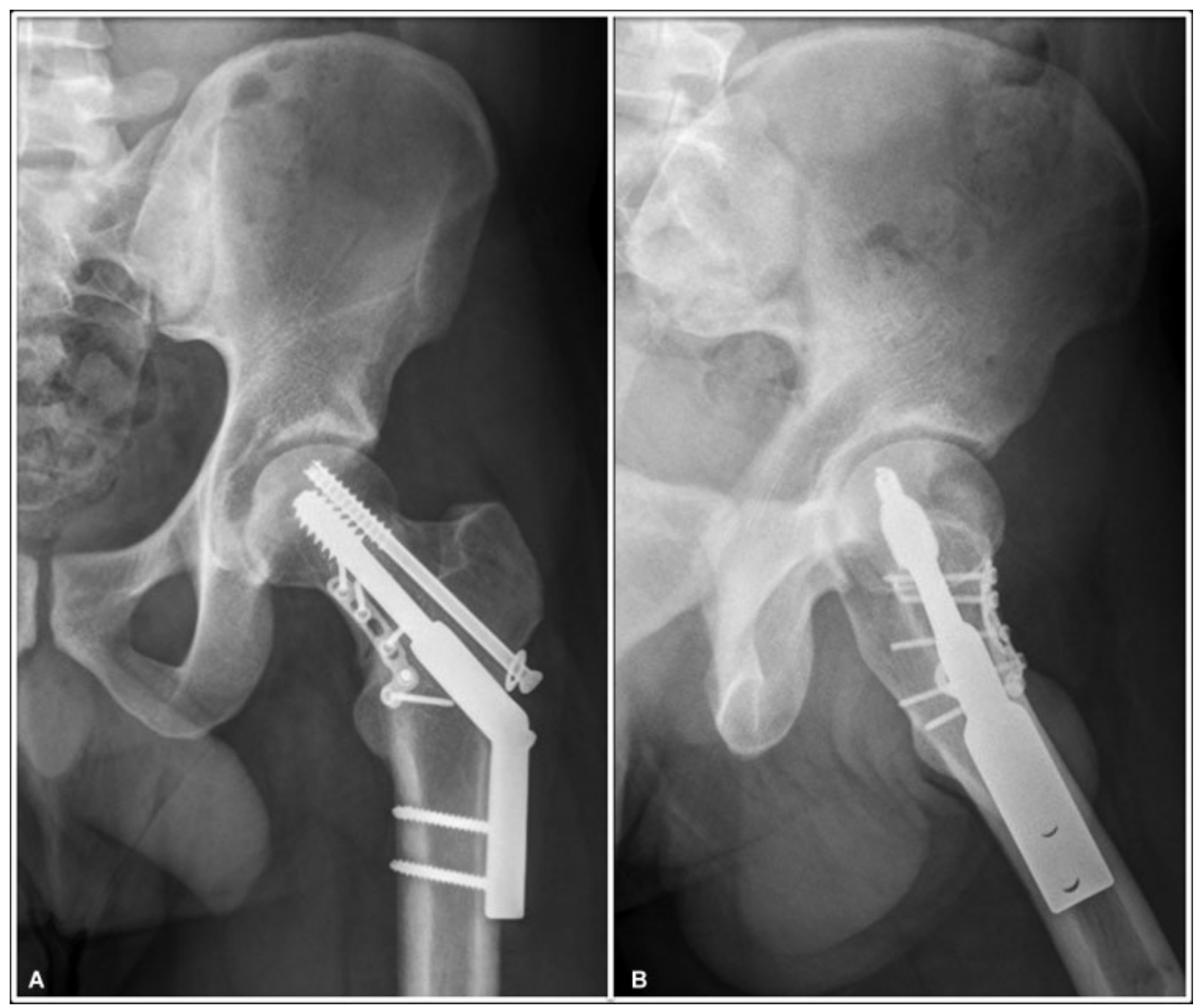

Fig. 7 (A) Radiografía de control del paciente de la - Fig. 6, 18 meses tras la osteosíntesis. Se realizó osteosíntesis con DHS $135^{\circ}$ de 2 orificios, tornillo antirrotatorio $7,0 \mathrm{~mm}$ y placa anterior en el cuello femoral $2,7 \mathrm{~mm}$. Se observa consolidación de la fractura con leve colapso sin evidencias de necrosis avascular. (B) Radiografía axial donde se evidencia como la placa anterior se moldea a la superficie del cuello femoral.

\section{Bibliografía}

1 Bhandari M, Tornetta P III, Hanson B, Swiontkowski MF. Optimal internal fixation for femoral neck fractures: multiple screws or sliding hip screws? J Orthop Trauma 2009;23(06):403-407

2 Gardner S, Weaver MJ, Jerabek S, Rodriguez E, Vrahas M, Harris M. Predictors of early failure in young patients with displaced femoral neck fractures. J Orthop 2014;12(02):75-80

3 Papakostidis C, Panagiotopoulos A, Piccioli A, Giannoudis PV. Timing of internal fixation of femoral neck fractures. A systematic review and meta-analysis of the final outcome. Injury 2015;46(03):459-466

4 Stacey SC, Renninger CH, Hak D, Mauffrey C. Tips and tricks for ORIF of displaced femoral neck fractures in the young adult patient. Eur J Orthop Surg Traumatol 2016;26(04):355-363

5 Kurylo JC, Templeman D, Mirick GE. The perfect reduction: approaches and techniques. Injury 2015;46(03):441-444

6 Probe R, Ward R. Internal fixation of femoral neck fractures. J Am Acad Orthop Surg 2006;14(09):565-571

7 Molnar RB, Routt ML Jr. Open reduction of intracapsular hip fractures using a modified Smith-Petersen surgical exposure. J Orthop Trauma 2007;21(07):490-494
8 Sheehan SE, Shyu JY, Weaver MJ, Sodickson AD, Khurana B. Proximal Femoral Fractures: What the Orthopedic Surgeon Wants to Know. Radiographics 2015;35(05):1563-1584

9 Slobogean GP, Sprague SA, Scott T, McKee M, Bhandari M. Management of young femoral neck fractures: is there a consensus? Injury 2015;46(03):435-440

10 Davidovitch RI, Jordan CJ, Egol KA, Vrahas MS. Challenges in the treatment of femoral neck fractures in the nonelderly adult. J Trauma 2010;68(01):236-242

11 Lowe JA, Crist BD, Bhandari M, Ferguson TA. Optimal treatment of femoral neck fractures according to patient's physiologic age: an evidence-based review. Orthop Clin North Am 2010;41(02): 157-166

12 Szita J, Cserháti P, Bosch U, Manninger J, Bodzay T, Fekete K. Intracapsular femoral neck fractures: the importance of early reduction and stable osteosynthesis. Injury 2002;33(03, Suppl 3): C41-C46

13 Maurer SG, Wright KE, Kummer FJ, Zuckerman JD, Koval KJ. Two or three screws for fixation of femoral neck fractures? Am J Orthop 2003;32(09):438-442 
14 Stockton DJ, Lefaivre KA, Deakin DE, et al. Incidence, Magnitude, and Predictors of Shortening in Young Femoral Neck Fractures. J Orthop Trauma 2015;29(09):e293-e298

15 Zlowodzki M, Brink O, Switzer J, et al. The effect of shortening and varus collapse of the femoral neck on function after fixation of intracapsular fracture of the hip: a multicentre cohort study. J Bone Joint Surg Br 2008;90(11): $1487-1494$
16 Lichstein PM, Kleimeyer JP, Githens M, et al. Does the WatsonJones or Modified Smith-Petersen Approach Provide Superior Exposure for Femoral Neck Fracture Fixation? Clin Orthop Relat Res 2018;476(07):1468-1476

17 Ye Y, Chen K, Tian K, Li W, Mauffrey C, Hak DJ. Medial buttress plate augmentation of cannulated screw fixation in vertically unstable femoral neck fractures: Surgical technique and preliminary results. Injury 2017;48(10):2189-2193 